\title{
Guidelines for imaging reach a new quality level towards computer-assisted diagnosis
}

\author{
Franz Kainberger
}

(C) Springer-Verlag GmbH Austria, part of Springer Nature 2018

For optimizing the transfer of clinical information to radiology departments, referral guidelines for imaging have been developed since the early 1990s. Their practical use has, however, been limited despite relevant progress in the design and worldwide support by radiological societies and governmental bodies. The reasons may be limitations in their design and implementation as well as the primary stakeholders' intentions to reduce costs and radiation exposure instead of focussing on the clinical needs. In this issue of the Wiener Klinische Wochenschrift-Central European Journal of Medicine, Tamandl et al. publish consensus guidelines for imaging of hepatic tumors [1]. Their design has the potential for overcoming these limitations by following new quality standards and by integrating the European level of knowledge.

Quality has been recently improved by the appraisal of guidelines for research and evaluation II (AGREE II) instrument which provides a grid for a transparent and critical evaluation of guidelines [2]. Additional quality aspects are related to safety, e.g. World Health Organization (WHO) patient safety initiative and European Union (EU) directives and to patient empowerment.

There is a field of tension between the best evidence and the local needs of a healthcare system and its resources, the epidemiology and society's expectations. A third dimension, and this might be a new aspect of guideline development, is the European one, as on this continent the local need is in most cases subject

\section{Prof. Dr. F. Kainberger}

Division of Neuro- and Musculoskeletal Radiology, Department of Biomedical Imaging and Image-Guided Therapy, Medical University of Vienna, Vienna, Austria to EU Council directives. They are, in turn, influenced by the diversity of national or other, sometimes conflicting recommendations.

The implementation of guidelines is generally underestimated compared to the efforts of their development. For imaging, they should be catalogued in lists of appropriateness criteria. With computer-assisted decision (CAD) support systems, highly efficient tools are available for bringing this content to the point-of-care, i.e. the referring physicians. Referrals are increasingly submitted in electronic form, and the key recommendations should be directly available in semi-automated CAD systems for supporting but not replacing the physician's referral [3].

In the upcoming new edition of the Austrian catalogue of referral guidelines these aspects will be realized [4]: (1) quality standards oriented to recent recommendations for guidelines development as well as to patients' needs and social outcomes, (2) referring to the three dimensions of best available evidence, local needs and European directives, (3) designed for practical use at the point-of-care with a print version and a clinical decision support tool and (4) based on the ground rules of diagnostic reasoning. This work is based on two decades of continuous refinements characterized by an increase in homogeneity of referral guidelines for imaging in Europe and by a significant increase in the volume of contents. As such, referral guidelines offer a concise thesaurus of what is possible with modern imaging.

Conflict of interest F. Kainberger declares that he has no competing interests.

\section{References}

1. Tamandl D, Ba-Ssalamah A, Böhm G, et al. Austrian consensus guidelines of imaging requirements prior to 


\section{editorial}

hepatic surgery and during follow-up in patients with malignant hepatic lesions. Wien Klin Wochenschr. 2018; https://doi.org/10.1007/s00508-018-1387-z.

2. Brouwers M, et al. Appraisal of Guidelines for Research and Evaluation II-AGREE II. 2017. http:/ / www.agreetrust.org/ wp-content/uploads/2013/10/AGREE-II-Users-Manualand-23-item-Instrument_2009_UPDATE_2013.pdf. Accessed 3 Aug 2018.
3. European Society of Radiology (ESR).. Summary of the proceedings of the international forum 2016: "Imaging referral guidelines and clinical decision support-how can radiologists implement imaging referral guidelines in clinical routine?". Insights Imaging. 2017;8(1):1-9.

4. Frühwald F, Tscholakoff D, Kainberger F, editors. Orientierungshilfe Radiologie. 5th ed. Vienna: Verlagshaus der Ärzte; 2011. 